\title{
Antirefluxchirurgie schützt nicht vor dem Ösophaguskarzinom
}

\author{
Ein Teil der Patienten \\ mit einer chronischen \\ Refluxkrankheit ent- \\ wickelt eine Barrett- \\ schleimhaut, die \\ wiederum als Präkan- \\ zerose für ein distales \\ Ösophaguskarzinom \\ gilt. Bisher gibt es \\ keine überzeugenden \\ Studienergebnisse \\ dahingehend, dass \\ durch eine konse- \\ quente hochdosierte
}

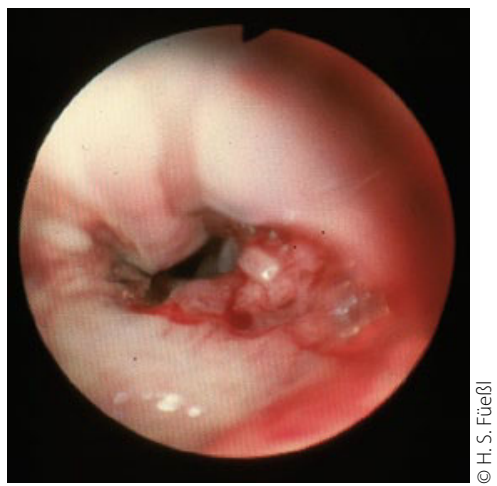

Ösophaguskarzinom: Bei Patienten mit Reflux zwölfmal häufiger.

Therapie mit einem

Protonenpumpenhemmer dieses Risiko beeinflusst werden kann. Gleiches dürfte nach einer neuen Studie auch für die Antirefluxchirurgie gelten.

- Analysiert wurden die Daten von 14102 Patienten, die sich einem antirefluxchirurgischen Eingriff, genauer gesagt einer laparaskopischen Fundoplikatio, unterzogen hatten. Bei insgesamt 39 Patienten entwickelte sich ein distales Ösophaguskarzinom. Damit war das Risiko zwölffach erhöht gegenüber Personen ohne Refluxkrankheit. Eine Beeinflussung des Risikos durch die Antirefluxchirurgie konnte während einer über 15-jährigen Verlaufsbeobachtung nicht dokumentiert werden.

\section{Kommentar}

Was für die Protonenpumpenhemmer gilt, trifft auch für die Antirefluxchirurgie zu: Die medikamentöse oder operative Behandlung der Refluxkrankheit ist eine symptomatische Therapie, ändert jedoch nichts am Entartungsrisiko. Somit ist es nicht gerechtfertigt, einem Patienten, der mit einem Protonenpumpenhemmer gut zurechtkommt, eine Fundoplikatio im Hinblick auf die Verhinderung eines Ösophaguskarzinoms zu empfehlen.

P. Stiefelhagen a

- J. Lagergen et al.

The risk of esophageal adenocarcinoma after antireflux surgery. Gastroenteroloy 138 (2010) 1297-1301 